\title{
Cyclic coverings of an elliptic curve with two branch points and the gap sequences at the ramification points
}

\author{
by \\ JiRYo KOMEDA (Atsugi)
}

1. Introduction. Let $C$ be a complete non-singular irreducible algebraic curve of genus $g \geq 2$ defined over an algebraically closed field $k$ of characteristic 0 , which is called a curve in this paper. Let $P$ be its point. A positive integer $\gamma$ is called a gap at $P$ if there exists a regular 1-form $\omega$ on $C$ such that $\operatorname{ord}_{P}(\omega)=\gamma-1$. We denote by $G(P)$ the set of gaps at $P$. Then the cardinality of $G(P)$ is equal to $g$. Now the sequence $\left\{\gamma_{1}, \ldots, \gamma_{g}\right\}=G(P)$ with $\gamma_{i}<\gamma_{j}$ for $i<j$ is called the gap sequence at $P$.

Let $\pi: C \rightarrow C^{\prime}$ be a cyclic covering of curves of degree $d$ with total ramification points $P$. It is well known that in the case of $C^{\prime}=\mathbb{P}^{1}$ and $d=2$ we have $G(P)=\{1,3, \ldots, 2 g-1\}$. In the case of $C^{\prime}=\mathbb{P}^{1}$ and $d=3$ (resp. 4) the gap sequences $G(P)$ are known (see [1], [2], [3] (resp. [4], Prop. 4.5)). If $C^{\prime}=\mathbb{P}^{1}$ and $d$ is a prime number $\geq 5$, we can also determine the gap sequences $G(P)$ (for example, see [5], Prop. 1). In this paper we shall consider the case $C^{\prime}=E$ where $E$ is an elliptic curve. If $d=2$, then $G(P)$ are known ([4], Prop. 2.9, 3.10). However, for $d \geq 3$ there are only a few results on the gap sequences $G(P)$. For example, I. Kuribayashi and K. Komiya ([8], Th. 5) showed the following: If $\pi: C \rightarrow E$ is a cyclic covering of an elliptic curve of degree 6 which is branched over three points $P_{i}^{\prime}(i=1,2,3)$ such that $\sharp \pi^{-1}\left(P_{i}^{\prime}\right)=i$, then the gap sequence $G\left(P_{1}\right)$ can be determined, where $P_{1}$ denotes the point of $C$ over $P_{1}^{\prime}$. Moreover, the author ([6], Lemma 4.6) showed the following: Let $E$ be an elliptic curve with the origin $Q^{\prime}$. Let $P_{1}^{\prime}$ (resp. $P_{2}^{\prime}$ ) be a point of $E$ such that $P_{1}^{\prime} \neq Q^{\prime}$ and $2\left[P_{1}^{\prime}\right]=\left[Q^{\prime}\right]\left(\right.$ resp. $P_{2}^{\prime} \neq Q^{\prime}$ and $\left.3\left[P_{2}^{\prime}\right]=\left[Q^{\prime}\right]\right)$, where for any positive integer $m$ and any point $P^{\prime}$ of the elliptic curve $E$ the multiplication of $P^{\prime}$ by $m$ is denoted by $m\left[P^{\prime}\right]$. Then there is an element $z$ of $\mathbf{K}(E)$ such

1991 Mathematics Subject Classification: Primary 14H55; Secondary 14H30, 14H52, $11 \mathrm{~A} 05$. 
that $\operatorname{div}(z)=4 P_{1}^{\prime}+3 P_{2}^{\prime}-7 Q^{\prime}$ where $\mathbf{K}(E)$ denotes the function field of $E$. Let $\pi: C \rightarrow E$ be the surjective morphism of curves corresponding to the inclusion $\mathbf{K}(E) \subset \mathbf{K}(E)\left(z^{1 / 7}\right)=\mathbf{K}(C)$. If $P_{2}$ denotes the point of $C$ over $P_{2}^{\prime}$, then the gap sequence $G\left(P_{2}\right)$ is equal to $\{1,2,3,4,5,7,13\}$. In this paper we shall prove the generalization of the above statement for the degree of the covering $\pi: C \rightarrow E$, which is the following:

Main Theorem. Let $g \geq 7$. We can construct cyclic coverings $\pi: C \rightarrow$ $E$ of an elliptic curve $E$ of degree $g$ which have only two ramification points $P_{1}$ and $P_{2}$, which are totally ramified, such that

$$
G\left(P_{1}\right)=G\left(P_{2}\right)=\{1, \ldots, g-2, g, 2 g-1\} .
$$

Now we consider the following situation. Let $G$ be a finite subset of the set $\mathbb{N}$ of positive integers such that the complement $\mathbb{N}_{0} \backslash G$ of $G$ in the additive semigroup $\mathbb{N}_{0}$ of non-negative integers forms its subsemigroup. If the cardinality of $G$ is $g$, then $\left\{\gamma_{1}, \ldots, \gamma_{g}\right\}=G$ with $\gamma_{i}<\gamma_{j}$ for $i<j$ is called a gap sequence of genus $g$. We say that a gap sequence $G$ is Weierstrass if there exists a pointed curve $(C, P)$ such that $G=G(P)$. Let $a(G)=\min \{h \in$ $\left.\mathbb{N}_{0} \backslash G \mid h>0\right\}$. Then $a(G) \leq g+1$. If $a(G)=g+1$, then $G=\{1, \ldots, g\}$. In this case $G$ is Weierstrass, because for any point $P$ of a curve of genus $g$ except finitely many points we have $G(P)=\{1, \ldots, g\}$. If $a(G)=g$, then there is a positive integer $k \leq g-1$ such that $G=\{1, \ldots, g-1, g+k\}$. These $g-1$ kinds of gap sequences are Weierstrass (cf. [9], Th. 14.5). If $l$ is a fixed integer $\geq 2$, then for any sufficiently large $g$ there exists a non-Weierstrass gap sequence $G$ of genus $g$ such that $a(G)=g-l$ (cf. [7], Th. 3.5 and 4.5). Hence we pose the following problem: Is any gap sequence $G$ of genus $g$ with $a(G)=g-1$ Weierstrass?

Now we say that $G$ is primitive if $2 a(G)>\gamma_{g}$. Since any gap sequence of genus $g \leq 7$ is Weierstrass (cf. [6], Th. 4.7), combining the Main Theorem with Lemma 1 we get the following:

Any non-primitive gap sequence $G$ of genus $g$ with $a(G)=g-1$ is Weierstrass.

In Sections 2, 3 and 4 we construct our desired cyclic coverings $\pi: C \rightarrow E$ of an elliptic curve in the cases when $g \equiv 3,1$ and $0 \bmod 4$ respectively. In Section 5 the case when $g \equiv 2 \bmod 4$ is treated. In this case we need an arithmetic lemma (Key Lemma 4) which is important for the constructions of the coverings $\pi: C \rightarrow E$.

2. The case $g \equiv 3 \bmod 4$. First we prove the following:

Lemma 1. Let $G$ be a non-primitive gap sequence of genus $g \geq 3$ with $a(G)=g-1$. Then $G=\{1, \ldots, g-2, g, 2 g-1\}$. 
Proof. Let $G=\left\{\gamma_{1}, \ldots, \gamma_{g}\right\}$ with $\gamma_{i}<\gamma_{j}$ for $i<j$. In view of $a(G)=$ $g-1$ we must have $\gamma_{i}=i$ for $i \leq g-2$ and $\gamma_{g-1} \geq g$. Since $G$ is non-primitive, we have $\gamma_{g}>2 a(G)=2 g-2$. It is a well-known fact that $\gamma_{g} \leq 2 g-1$ (for example, see [4], Lemma 2.1), which implies that $\gamma_{g}=2 g-1$. Suppose that $\gamma_{g-1} \geq g+1$. Then $\mathbb{N}_{0} \backslash G$ contains $g-1$ and $g$. Since $\mathbb{N}_{0} \backslash G$ is a subsemigroup of $\mathbb{N}_{0}$, we must have $\gamma_{g}=2 g-1 \in \mathbb{N}_{0} \backslash G$, which is a contradiction. Hence we obtain $\gamma_{g-1}=g$.

In the remainder of this section we will prove the Main Theorem in the case $g \equiv 3 \bmod 4$ with $g \geq 7$.

Let $g=4 h+3=2 n+1$ with $h \in \mathbb{N}$ and $n=2 h+1$. Let $E$ be an elliptic curve over $k$ with the origin $Q^{\prime}$. Let $P_{1}^{\prime}$ be a point of $E$ such that $P_{1}^{\prime} \neq Q^{\prime}$ and $2\left[P_{1}^{\prime}\right]=\left[Q^{\prime}\right]$. Moreover, $P_{2}^{\prime}$ denotes a point of $E$ such that $n\left[P_{2}^{\prime}\right]=\left[Q^{\prime}\right]$ and $m\left[P_{2}^{\prime}\right] \neq\left[Q^{\prime}\right]$ for any positive integer $m<n$. Hence in view of $g \geq 7$ we have $P_{2}^{\prime} \neq Q^{\prime}$. Moreover, $P_{1}^{\prime} \neq P_{2}^{\prime}$, because $2 h P_{2}^{\prime}+P_{2}^{\prime}=n P_{2}^{\prime} \sim n Q^{\prime}=$ $(2 h+1) Q^{\prime} \sim 2 h P_{1}^{\prime}+Q^{\prime}$. Now we have

$$
(n+1) P_{1}^{\prime}+n P_{2}^{\prime} \sim 2(h+1) P_{1}^{\prime}+n Q^{\prime} \sim 2(h+1) Q^{\prime}+n Q^{\prime}=(2 n+1) Q^{\prime} .
$$

Hence we may take $z \in \mathbf{K}(E)$ such that $\operatorname{div}(z)=(n+1) P_{1}^{\prime}+n P_{2}^{\prime}-(2 n+1) Q^{\prime}$.

Let $C$ be the curve whose function field $\mathbf{K}(C)$ is $\mathbf{K}(E)\left(z^{1 /(2 n+1)}\right)$. Moreover, $\pi: C \rightarrow E$ denotes the surjective morphism of curves corresponding to the inclusion $\mathbf{K}(E) \subset \mathbf{K}(C)$. Then we may take $y \in \mathbf{K}(C)$ and $\sigma \in \operatorname{Aut}(\mathbf{K}(C) / \mathbf{K}(E))$ such that

$$
\sigma(y)=\zeta_{2 n+1} y \quad \text { and } \quad \operatorname{div}_{E}\left(y^{2 n+1}\right)=(n+1) P_{1}^{\prime}+n P_{2}^{\prime}-(2 n+1) Q^{\prime},
$$

where $\zeta_{2 n+1}$ is a primitive $(2 n+1)$ th root of unity. Then there are only two branch points $P_{1}^{\prime}$ and $P_{2}^{\prime}$ of $\pi$. Moreover, $\pi^{-1}\left(P_{i}^{\prime}\right)$ consists of only one point $P_{i}$ for $i=1,2$. Hence the ramification index of $P_{i}$ is $2 n+1$ for $i=1,2$. Therefore

$$
\operatorname{div}(y)=(n+1) P_{1}+n P_{2}-\pi^{*}\left(Q^{\prime}\right),
$$

where $\pi^{*}$ denotes the pull-back of $\pi$. If we denote by $g$ the genus of $C$, then by the Riemann-Hurwitz formula we have $g=2 n+1$. Hence

$$
\operatorname{div}(d y)=n P_{1}+(n-1) P_{2}-2 \pi^{*}\left(Q^{\prime}\right)+\sum_{i=1}^{3} \pi^{*}\left(R_{i}^{\prime}\right),
$$

where $R_{i}^{\prime}$ 's are points of $E$ which are distinct from $P_{1}^{\prime}, P_{2}^{\prime}$ and $Q^{\prime}$, because $\operatorname{div}(d y)$ is invariant under $\operatorname{Aut}(\mathbf{K}(C) / \mathbf{K}(E))$.

We set

$$
D_{0}^{\prime}=-P_{1}^{\prime}-P_{2}^{\prime}-Q^{\prime}+\sum_{i=1}^{3} R_{i}^{\prime}
$$




$$
D_{2 l+1}^{\prime}=-(2 l+2) Q^{\prime}+l P_{1}^{\prime}+l P_{2}^{\prime}+\sum_{i=1}^{3} R_{i}^{\prime} \quad \text { for } 0 \leq l \leq n-1
$$

and

$$
D_{2 l}^{\prime}=-(2 l+1) Q^{\prime}+l P_{1}^{\prime}+(l-1) P_{2}^{\prime}+\sum_{i=1}^{3} R_{i}^{\prime} \quad \text { for } 1 \leq l \leq n .
$$

First we show that $l\left(D_{0}^{\prime}\right)=1$, i.e., $D_{0}^{\prime}$ is linearly equivalent to 0 , where for any divisor $D^{\prime}$ on $E$ the number $l\left(D^{\prime}\right)$ denotes the dimension of the $k$-vector space

$$
L\left(D^{\prime}\right)=\left\{f \in \mathbf{K}(E) \mid \operatorname{div}_{E}(f) \geq-D^{\prime}\right\} .
$$

Since

$$
\sigma\left(\frac{d y}{y}\right)=\frac{d(\sigma y)}{\sigma y}=\frac{d\left(\zeta_{2 n+1} y\right)}{\zeta_{2 n+1} y}=\frac{d y}{y}
$$

the 1-form $d y / y$ on $C$ is regarded as the one on $E$. Hence there exists an element $f$ of $\mathbf{K}(E)$ such that $f d y / y$ is regular. Then

$$
\operatorname{div}_{E}(f)=P_{1}^{\prime}+P_{2}^{\prime}+Q^{\prime}-\sum_{i=1}^{3} R_{i}^{\prime}
$$

because

$$
\begin{aligned}
0 & \leq \operatorname{div}_{C}\left(\frac{f d y}{y}\right)=\operatorname{div}_{C}(f)+\operatorname{div}_{C}\left(\frac{d y}{y}\right) \\
& =\operatorname{div}_{C}(f)-P_{1}-P_{2}-\pi^{*}\left(Q^{\prime}\right)+\sum_{i=1}^{3} \pi^{*}\left(R_{i}^{\prime}\right)
\end{aligned}
$$

Hence

$$
D_{0}^{\prime}=-P_{1}^{\prime}-P_{2}^{\prime}-Q^{\prime}+\sum_{i=1}^{3} R_{i}^{\prime} \sim 0 .
$$

Moreover, $l\left(D_{r}^{\prime}\right)=1$ for any $r$ with $1 \leq r \leq 2 n$, because $\operatorname{deg}\left(D_{r}^{\prime}\right)=1$.

To compute the numbers $l\left(D_{r}^{\prime}-P_{1}^{\prime}\right)$ and $l\left(D_{r}^{\prime}-P_{2}^{\prime}\right)$ we show that $m P_{1}^{\prime} \chi$ $m P_{2}^{\prime}$ for any positive integer $m$ with $m \leq n$. In fact, suppose that there exists a positive integer $m \leq n$ such that $m P_{1}^{\prime} \sim m P_{2}^{\prime}$. If $m$ is even, then

$$
m P_{2}^{\prime} \sim \frac{m}{2} 2 P_{1}^{\prime} \sim \frac{m}{2} 2 Q^{\prime}=m Q^{\prime},
$$

which is a contradiction. Let $m$ be odd. Then $2 m P_{2}^{\prime} \sim 2 m P_{1}^{\prime} \sim 2 m Q^{\prime}$. If $m<n / 2$, then

$$
(n-2 m) P_{2}^{\prime}=n P_{2}^{\prime}-2 m P_{2}^{\prime} \sim n Q^{\prime}-2 m Q^{\prime}=(n-2 m) Q^{\prime},
$$


a contradiction. If $n / 2<m<n$, then $(2 m-n) P_{2}^{\prime} \sim(2 m-n) Q^{\prime}$, a contradiction. If $m=n$, then

$$
(n-1) Q^{\prime}+P_{1}^{\prime} \sim(n-1) P_{1}^{\prime}+P_{1}^{\prime} \sim n P_{2}^{\prime} \sim n Q^{\prime},
$$

which implies that $P_{1}^{\prime} \sim Q^{\prime}$. This is a contradiction. Hence we have shown that for any $m$ with $0<m \leq n, m P_{1}^{\prime} \not m P_{2}^{\prime}$.

Now for any $l$ with $0 \leq l \leq n-2$ we have $l\left(D_{2 l+1}^{\prime}-P_{1}^{\prime}\right)=0$. In fact, suppose that $l\left(D_{2 l+1}^{\prime}-P_{1}^{\prime}\right)=1$. Then

$$
\begin{aligned}
0 & \sim D_{2 l+1}^{\prime}-P_{1}^{\prime}-D_{0}^{\prime} \sim(n-2 l-1) Q^{\prime}+l P_{1}^{\prime}+(l+1-n) P_{2}^{\prime} \\
& \sim(n-l-1) P_{1}^{\prime}-(n-l-1) P_{2}^{\prime},
\end{aligned}
$$

because $n Q^{\prime} \sim n P_{2}^{\prime}$ and $2 P_{1}^{\prime} \sim 2 Q^{\prime}$. Hence

$$
1 \leq n-l-1 \leq n-1 \text { and }(n-l-1) P_{1}^{\prime} \sim(n-l-1) P_{2}^{\prime},
$$

which is a contradiction.

Now in view of $2 P_{1}^{\prime} \sim 2 Q^{\prime}$ and $n P_{2}^{\prime} \sim n Q^{\prime}$ we have

$$
D_{2 n-1}^{\prime}-P_{1}^{\prime}-D_{0}^{\prime} \sim-(2 n-1) Q^{\prime}+(n-1) Q^{\prime}+n Q^{\prime}=0,
$$

which implies that $D_{2 n-1}^{\prime}-P_{1}^{\prime} \sim 0$. Hence

$$
l\left(D_{2 n-1}^{\prime}\right)=l\left(D_{2 n-1}^{\prime}-P_{1}^{\prime}\right)=1 \quad \text { and } \quad l\left(D_{2 n-1}^{\prime}-2 P_{1}^{\prime}\right)=0 .
$$

Suppose that $l\left(D_{2 l}^{\prime}-P_{1}^{\prime}\right)=1$. Then in view of $2 P_{1}^{\prime} \sim 2 Q^{\prime}$ we have

$$
0 \sim D_{2 l}^{\prime}-P_{1}^{\prime}-D_{0}^{\prime} \sim-2 l P_{1}^{\prime}+l P_{1}^{\prime}+l P_{2}^{\prime}=-l P_{1}^{\prime}+l P_{2}^{\prime},
$$

a contradiction. Hence $l\left(D_{2 l}^{\prime}-P_{1}^{\prime}\right)=0$ for any $l$ with $1 \leq l \leq n$.

Next we show that $l\left(D_{1}^{\prime}-P_{2}^{\prime}\right)=0$. If $l\left(D_{1}^{\prime}-P_{2}^{\prime}\right)=1$, then

$$
-2 Q^{\prime}+\sum_{i=1}^{3} R_{i}^{\prime}-P_{2}^{\prime}=D_{1}^{\prime}-P_{2}^{\prime} \sim 0 \sim D_{0}^{\prime} \sim-P_{1}^{\prime}-P_{2}^{\prime}-Q^{\prime}+\sum_{i=1}^{3} R_{i}^{\prime},
$$

which implies that $P_{1}^{\prime} \sim Q^{\prime}$. This is a contradiction. Now in view of $2 P_{1}^{\prime} \sim$ $2 Q^{\prime}$ we obtain $D_{2}^{\prime}-P_{2}^{\prime} \sim D_{0}^{\prime} \sim 0$, which implies that

$$
l\left(D_{2}^{\prime}\right)=l\left(D_{2}^{\prime}-P_{2}^{\prime}\right)=1 \text { and } l\left(D_{2}^{\prime}-2 P_{2}^{\prime}\right)=0 .
$$

Let $1 \leq l \leq n-1$. Suppose that $l\left(D_{2 l+1}^{\prime}-P_{2}^{\prime}\right)=1$. Then

$$
-(2 l+2) Q^{\prime}+l P_{1}^{\prime}+(l-1) P_{2}^{\prime}+\sum_{i=1}^{3} R_{i}^{\prime} \sim D_{0}^{\prime} \sim-P_{1}^{\prime}-P_{2}^{\prime}-Q^{\prime}+\sum_{i=1}^{3} R_{i}^{\prime},
$$

which implies that $-(l+1) P_{1}^{\prime} \sim-(2 l+1) Q^{\prime}+l P_{2}^{\prime}$. Since $n P_{2}^{\prime} \sim n Q^{\prime}$ and $n$ is odd, we have

$$
n P_{2}^{\prime}-(l+1) P_{1}^{\prime} \sim(n-(2 l+1)) Q^{\prime}+l P_{2}^{\prime} \sim(n-(2 l+1)) P_{1}^{\prime}+l P_{2}^{\prime},
$$

which implies that $(n-l) P_{2}^{\prime} \sim(n-l) P_{1}^{\prime}$. This contradicts $m P_{1}^{\prime} \nsim m P_{2}^{\prime}$ for any $0<m<n$. Hence $l\left(D_{2 l+1}^{\prime}-P_{2}^{\prime}\right)=0$ for any $1 \leq l \leq n-1$. 
Let $2 \leq l \leq n$. Suppose that $l\left(D_{2 l}^{\prime}-P_{2}^{\prime}\right)=1$. Then

$$
-(2 l+1) Q^{\prime}+l P_{1}^{\prime}+(l-2) P_{2}^{\prime}+\sum_{i=1}^{3} R_{i}^{\prime} \sim-P_{1}^{\prime}-P_{2}^{\prime}-Q^{\prime}+\sum_{i=1}^{3} R_{i}^{\prime},
$$

which implies that $(l+1) P_{1}^{\prime}+(l-1) P_{2}^{\prime} \sim 2 l Q^{\prime} \sim 2 l P_{1}^{\prime}$. Hence $(l-1) P_{2}^{\prime} \sim$ $(l-1) P_{1}^{\prime}$, a contradiction. Therefore $l\left(D_{2 l}^{\prime}-P_{2}^{\prime}\right)=0$ for any $2 \leq l \leq n$.

Now let $f$ be an element of $\mathbf{K}(E)$ and set

$$
\operatorname{div}_{E}(f)=\sum_{P^{\prime} \in E} m\left(P^{\prime}\right) P^{\prime}
$$

Then for any non-negative integer $r$ we obtain

$$
\begin{aligned}
\operatorname{div}_{C}\left(\frac{f d y}{y^{1-r}}\right)= & \left((2 n+1) m\left(P_{1}^{\prime}\right)+n+(n+1)(r-1)\right) P_{1} \\
& +\left((2 n+1) m\left(P_{2}^{\prime}\right)+n-1+n(r-1)\right) P_{2} \\
& +\left(m\left(Q^{\prime}\right)-r-1\right) \pi^{*}\left(Q^{\prime}\right) \\
& +\sum_{i=1}^{3}\left(m\left(R_{i}^{\prime}\right)+1\right) \pi^{*}\left(R_{i}^{\prime}\right)+\sum_{P^{\prime} \in S} m\left(P^{\prime}\right) \pi^{*}\left(P^{\prime}\right),
\end{aligned}
$$

where we set $S=E \backslash\left\{P_{1}^{\prime}, P_{2}^{\prime}, Q^{\prime}, R_{1}^{\prime}, R_{2}^{\prime}, R_{3}^{\prime}\right\}$. We note that if $R_{1}^{\prime} \neq R_{2}^{\prime}$ and $R_{2}^{\prime}=R_{3}^{\prime}$ (resp. $R_{1}^{\prime}=R_{2}^{\prime}=R_{3}^{\prime}$ ), then

$$
\sum_{i=1}^{3}\left(m\left(R_{i}^{\prime}\right)+1\right) \pi^{*}\left(R_{i}^{\prime}\right)
$$

is replaced by

$$
\left(m\left(R_{1}^{\prime}\right)+1\right) \pi^{*}\left(R_{1}^{\prime}\right)+\left(m\left(R_{2}^{\prime}\right)+2\right) \pi^{*}\left(R_{2}^{\prime}\right) \quad\left(\text { resp. }\left(m\left(R_{1}^{\prime}\right)+3\right) \pi^{*}\left(R_{1}^{\prime}\right)\right) .
$$

For each $r=0,1, \ldots, 2 n$, we take a non-zero element $f_{r} \in L\left(D_{r}^{\prime}\right)$ and set $\phi_{r}=f_{r} d y / y^{1-r}$. Then by the above,

$$
\operatorname{ord}_{P_{i}}\left(\phi_{0}\right)=2 n+1-1=g-1 \quad \text { for } i=1,2 .
$$

For any $l$ with $0 \leq l \leq n-2$ we have

$$
\operatorname{ord}_{P_{1}}\left(\phi_{2 l+1}\right)=n+l+1-1 \quad \text { and } \quad \operatorname{ord}_{P_{2}}\left(\phi_{2 l+1}\right)=n-l-1 .
$$

Let $l=n-1$, i.e., $2 l+1=2 n-1$. Since $L\left(D_{2 n-1}^{\prime}\right)=L\left(D_{2 n-1}^{\prime}-P_{1}^{\prime}\right)$ and $L\left(D_{2 n-1}^{\prime}\right) \supset L\left(D_{2 n-1}^{\prime}-P_{2}^{\prime}\right)=(0)$, we obtain

$$
\operatorname{ord}_{P_{1}}\left(\phi_{2 n-1}\right)=4 n+1-1=2 g-1-1 \quad \text { and } \operatorname{ord}_{P_{2}}\left(\phi_{2 n-1}\right)=1-1 .
$$

Let $l=1$, i.e., $2 l=2$. Since $L\left(D_{2}^{\prime}\right) \supset L\left(D_{2}^{\prime}-P_{1}^{\prime}\right)=(0)$ and $L\left(D_{2}^{\prime}\right)=$ $L\left(D_{2}^{\prime}-P_{2}^{\prime}\right)$, we obtain

$$
\operatorname{ord}_{P_{1}}\left(\phi_{2}\right)=1-1 \quad \text { and } \operatorname{ord}_{P_{2}}\left(\phi_{2}\right)=2 g-1-1 .
$$


For any $l$ with $2 \leq l \leq n$ we have

$$
\operatorname{ord}_{P_{1}}\left(\phi_{2 l}\right)=l-1 \quad \text { and } \quad \operatorname{ord}_{P_{2}}\left(\phi_{2 l}\right)=2 n-l+1-1 .
$$

Hence for each $r=0,1, \ldots, 2 n, \phi_{r}$ is a regular 1 -form on $C$. Therefore $G\left(P_{1}\right)=G\left(P_{2}\right)=\{1, \ldots, g-2, g, 2 g-1\}$.

3. The case $g \equiv 1 \bmod 4$. In this section we prove the Main Theorem in the case $g \equiv 1 \bmod 4$ with $g \geq 9$.

Let $g=4 h+1=2 n+1$ with $h \in \mathbb{N}, h \geq 2$ and $n=2 h$. Let $E$ be an elliptic curve over $k$ with the origin $Q^{\prime}$. Let $P_{1}^{\prime}$ be a point of $E$ such that $P_{1}^{\prime} \neq Q^{\prime}$ and $2\left[P_{1}^{\prime}\right]=\left[Q^{\prime}\right]$. Moreover, $P_{2}^{\prime}$ denotes a point of $E$ such that $n\left[P_{2}^{\prime}\right]=-\left[P_{1}^{\prime}\right]$ and $m\left[P_{2}^{\prime}\right] \neq-\left[P_{1}^{\prime}\right]$ for any positive integer $m<n$, where $-\left[P_{1}^{\prime}\right]$ denotes the inverse of $P_{1}^{\prime}$ under the addition on the elliptic curve $E$. Then $P_{2}^{\prime} \neq Q^{\prime}$ and $P_{1}^{\prime} \neq P_{2}^{\prime}$. Moreover, $(n+1) P_{1}^{\prime}+n P_{2}^{\prime} \sim n Q^{\prime}+P_{1}^{\prime}+(n+1) Q^{\prime}-P_{1}^{\prime}=(2 n+1) Q^{\prime}$. Hence we may take $z \in \mathbf{K}(E) \operatorname{such}$ that $\operatorname{div}(z)=(n+1) P_{1}^{\prime}+n P_{2}^{\prime}-(2 n+1) Q^{\prime}$.

Let $C, \pi: C \rightarrow E, y \in \mathbf{K}(C), P_{1}, P_{2}, R_{i}^{\prime}, D_{0}^{\prime}, D_{2 l+1}^{\prime}$ and $D_{2 l}^{\prime}$ be as in Section 2. Then, in the same way as in Section $2, D_{0}^{\prime}$ is linearly equivalent to zero. Moreover, $l\left(D_{r}^{\prime}\right)=1$ for any $r$ with $1 \leq r \leq 2 n$.

To compute the numbers $l\left(D_{r}^{\prime}-P_{1}^{\prime}\right)$ and $l\left(D_{r}^{\prime}-P_{2}^{\prime}\right)$ we show that for any positive integer $m$ with $m \leq n, m P_{1}^{\prime} \not m P_{2}^{\prime}$. In fact, suppose that there exists a positive integer $m \leq n$ such that $m P_{1}^{\prime} \sim m P_{2}^{\prime}$. If $m$ is odd, then $m P_{2}^{\prime}+P_{1}^{\prime} \sim(m+1) P_{1}^{\prime} \sim(m+1) Q^{\prime}$. This contradicts $m\left[P_{2}^{\prime}\right] \neq-\left[P_{1}^{\prime}\right]$ for any positive integer $m<n$. If $m$ is even, then

$$
\begin{aligned}
(n+1) Q^{\prime} & \sim n P_{2}^{\prime}+P_{1}^{\prime}=(n-m) P_{2}^{\prime}+P_{1}^{\prime}+m P_{2}^{\prime} \\
& \sim(n-m) P_{2}^{\prime}+P_{1}^{\prime}+m P_{1}^{\prime} \sim(n-m) P_{2}^{\prime}+P_{1}^{\prime}+m Q^{\prime},
\end{aligned}
$$

which implies that $(n-m) P_{2}^{\prime}+P_{1}^{\prime} \sim(n+1-m) Q^{\prime}$. This is a contradiction.

For any $l$ with $0 \leq l \leq n-2$ we have $l\left(D_{2 l+1}^{\prime}-P_{1}^{\prime}\right)=0$. In fact, suppose that $l\left(D_{2 l+1}^{\prime}-P_{1}^{\prime}\right)=1$. Then $0 \sim D_{2 l+1}^{\prime}-P_{1}^{\prime}-D_{0}^{\prime}=-(2 l+1) Q^{\prime}+l P_{1}^{\prime}+$ $(l+1) P_{2}^{\prime}$. Since $n P_{2}^{\prime}+P_{1}^{\prime} \sim(n+1) Q^{\prime}$ and $n$ is even, we have

$$
\begin{aligned}
n P_{2}^{\prime}-l P_{1}^{\prime} & \sim-P_{1}^{\prime}+(n+1) Q^{\prime}-(2 l+1) Q^{\prime}+(l+1) P_{2}^{\prime} \\
& =-P_{1}^{\prime}+(l+1) P_{2}^{\prime}+(n-2 l) Q^{\prime} \sim-P_{1}^{\prime}+(l+1) P_{2}^{\prime}+(n-2 l) P_{1}^{\prime},
\end{aligned}
$$

which implies that $(n-l-1) P_{2}^{\prime} \sim(n-l-1) P_{1}^{\prime}$. This contradicts $m P_{1}^{\prime} \nsim m P_{2}^{\prime}$ for $1 \leq m \leq n$. Since $n P_{2}^{\prime}+P_{1}^{\prime} \sim(n+1) Q^{\prime}$ and $n$ is even, we have

$$
\begin{aligned}
D_{2 n-1}^{\prime}-P_{1}^{\prime}-D_{0}^{\prime} & \sim-(2 n-1) Q^{\prime}+(n-1) P_{1}^{\prime}+n P_{2}^{\prime} \\
& =-(n-2) Q^{\prime}+(n-2) P_{1}^{\prime} \sim-(n-2) Q^{\prime}+(n-2) Q^{\prime}=0,
\end{aligned}
$$

which implies that $l\left(D_{2 n-1}^{\prime}\right)=1=l\left(D_{2 n-1}^{\prime}-P_{1}^{\prime}\right)$. Moreover, in the same way as in Section 2 , we obtain $l\left(D_{2 l}^{\prime}-P_{1}^{\prime}\right)=0$ for any $l$ with $1 \leq l \leq n$.

Next, as in Section 2, we have

$$
l\left(D_{1}^{\prime}-P_{2}^{\prime}\right)=0 \quad \text { and } \quad l\left(D_{2}^{\prime}\right)=l\left(D_{2}^{\prime}-P_{2}^{\prime}\right)=1 .
$$


Let $1 \leq l \leq n-1$. Suppose $l\left(D_{2 l+1}^{\prime}-P_{2}^{\prime}\right)=1$. Then $D_{2 l+1}^{\prime}-P_{2}^{\prime} \sim 0$ $\sim D_{0}^{\prime}$, which implies that $-(l+1) P_{1}^{\prime} \sim-(2 l+1) Q^{\prime}+l P_{2}^{\prime}$. Since $n P_{2}^{\prime}+P_{1}^{\prime}$ $\sim(n+1) Q^{\prime}$ and $n$ is even, we have $n P_{2}^{\prime}-l P_{1}^{\prime} \sim(n+1) Q^{\prime}-(2 l+1) Q^{\prime}+l P_{2}^{\prime}$ $\sim(n-2 l) P_{1}^{\prime}+l P_{2}^{\prime}$, which implies that $(n-l) P_{2}^{\prime} \sim(n-l) P_{1}^{\prime}$. This is a contradiction. Hence $l\left(D_{2 l+1}^{\prime}-P_{2}^{\prime}\right)=0$ for any $1 \leq l \leq n-1$.

As in Section 2 we have $l\left(D_{2 l}^{\prime}-P_{2}^{\prime}\right)=0$ for any $2 \leq l \leq n$. Therefore $G\left(P_{1}\right)=G\left(P_{2}\right)=\{1, \ldots, g-2, g, 2 g-1\}$.

4. The case $g \equiv 0 \bmod 4$. First we show the following lemma, which is useful to construct the desired coverings of an elliptic curve in the even genus cases.

Lemma 2. Let $\pi_{0}: C \rightarrow C_{0}$ be a finite morphism of curves of degree 2 . Let $P \in C$ be a ramification point of $\pi_{0}$. Then $n \in \mathbb{N}_{0} \backslash G\left(\pi_{0}(P)\right)$ if and only if $2 n \in \mathbb{N}_{0} \backslash G(P)$.

Proof. Suppose that $n \in \mathbb{N}_{0} \backslash G\left(\pi_{0}(P)\right)$, i.e., there exists $f_{0} \in \mathbf{K}\left(C_{0}\right)$ such that $\left(f_{0}\right)_{\infty}=n \pi_{0}(P)$, where $\left(f_{0}\right)_{\infty}$ denotes the polar divisor of $f_{0}$. Since $P$ is a ramification point of $\pi_{0}$, we have $\left(\pi_{0}^{*} f_{0}\right)_{\infty}=2 n P$, where $\pi_{0}^{*}$ denotes the inclusion map $\mathbf{K}\left(C_{0}\right) \subset \mathbf{K}(C)$ corresponding to the surjective morphism $\pi_{0}: C \rightarrow C_{0}$. Hence $2 n \in \mathbb{N}_{0} \backslash G(P)$.

Conversely, suppose that $2 n \in \mathbb{N}_{0} \backslash G(P)$, i.e., there exists $f \in \mathbf{K}(C)$ such that $(f)_{\infty}=2 n P$. Let $\sigma$ be an involution of $C$ such that $C /\langle\sigma\rangle \cong C_{0}$. Then we may take a local parameter $t$ at $P$ such that $\sigma^{*} t=-t$. Since we can write

$$
f=c_{-2 n} t^{-2 n}+c_{-2 n+1} t^{-2 n+1}+\ldots
$$

where $c_{-2 n}$ is a non-zero constant and $c_{i}$ 's $(i \geq-2 n+1)$ are constants, we obtain

$$
\sigma^{*} f=c_{-2 n} t^{-2 n}-c_{-2 n+1} t^{-2 n+1}+\ldots
$$

Hence

$$
f+\sigma^{*} f=2 c_{-2 n} t^{-2 n}+2 c_{-2 n+2} t^{-2 n+2}+\ldots,
$$

which implies that $\left(f+\sigma^{*} f\right)_{\infty}=2 n P$. Now

$$
\sigma^{*}\left(f+\sigma^{*} f\right)=\sigma^{*} f+\left(\sigma^{2}\right)^{*} f=f+\sigma^{*} f,
$$

which implies that $f+\sigma^{*} f \in \mathbf{K}\left(C_{0}\right)$. Therefore $\left(f+\sigma^{*} f\right)_{\infty}=n \pi_{0}(P)$ on $C_{0}$, which implies that $n \in \mathbb{N}_{0} \backslash G\left(\pi_{0}(P)\right)$.

Using the above lemma we get the following:

Proposition 3. Let $\pi_{0}: C \rightarrow C_{0}$ be a finite morphism of curves of degree 2. Suppose that the genus $g$ of $C$ is even and that the genus of $C_{0}$ is equal to $\mathrm{g} / 2$. Let $P \in C$ be a ramification point of $\pi_{0}$. If $G(P)$ contains $\{2,4, \ldots, g-2, g, 2 g-1\}$, then $G(P)=\{1,2, \ldots, g-2, g, 2 g-1\}$. 
Pr o of. Suppose that $G(P) \supset\{2,4, \ldots, g-2, g, 2 g-1\}$. Then by Lemma 2 we obtain

$$
G\left(\pi_{0}(P)\right)=\{1,2, \ldots, g / 2\} .
$$

If $h$ is an even integer $>g$, then by the above we have $h / 2 \in \mathbb{N}_{0} \backslash G\left(\pi_{0}(P)\right)$. Hence by Lemma 2 we get $h \in \mathbb{N}_{0} \backslash G(P)$. On the other hand, if $h$ is an even integer with $g+2 \leq h \leq 2 g-2$, then $2 g-1-h \in G(P)$. In fact, if $2 g-1-h \in \mathbb{N}_{0} \backslash G(P)$, then $2 g-1=h+(2 g-1-h) \in \mathbb{N}_{0} \backslash G(P)$, a contradiction. Hence $G(P)$ contains the set

$\{2,4, \ldots, g-2, g, 2 g-1\} \cup\{2 g-1-h \mid h$ is even with $g+2 \leq h \leq 2 g-2\}$ $=\{1,2,3,4, \ldots, g-3, g-2, g, 2 g-1\}$.

Since the cardinality of $G(P)$ is $g$, we get the desired result.

Using this result we show the Main Theorem in the case $g \equiv 0 \bmod 4$ with $g \geq 8$.

Let $g=4 h=2 n$ with $h \in \mathbb{N}, h \geq 2$ and $n=2 h$. Let $E$ be an elliptic curve over $k$ with the origin $Q^{\prime}$. Let $P_{1}^{\prime}$ be a point of $E$ such that $(2 n-1)\left[P_{1}^{\prime}\right]=\left[Q^{\prime}\right]$ and $m\left[P_{1}^{\prime}\right] \neq\left[Q^{\prime}\right]$ for any positive integer $m<2 n-1$. Moreover, $P_{2}^{\prime}$ denotes the point of $E$ such that $\left[P_{2}^{\prime}\right]=3\left[P_{1}^{\prime}\right]$. Then $P_{2}^{\prime} \neq Q^{\prime}$ and $P_{1}^{\prime} \neq P_{2}^{\prime}$ because $g \geq 8$. Now we have

$$
\begin{aligned}
(n+1) P_{1}^{\prime}+(n-1) P_{2}^{\prime} & \sim(n+1) P_{1}^{\prime}+(n-1)\left(3 P_{1}^{\prime}-2 Q^{\prime}\right) \\
& \sim 2(2 n-1) P_{1}^{\prime}-(2 n-2) Q^{\prime} \sim 2 n Q^{\prime} .
\end{aligned}
$$

Hence we may take $z \in \mathbf{K}(E)$ such that $\operatorname{div}(z)=(n+1) P_{1}^{\prime}+(n-1) P_{2}^{\prime}-2 n Q^{\prime}$.

Let $C$ be the curve whose function field $\mathbf{K}(C)$ is $\mathbf{K}(E)\left(z^{1 /(2 n)}\right)$. Moreover, $\pi: C \rightarrow E$ denotes the surjective morphism of curves corresponding to the inclusion $\mathbf{K}(E) \subset \mathbf{K}(C)$. Then we may take $y \in \mathbf{K}(C)$ and $\sigma \in \operatorname{Aut}(\mathbf{K}(C) / \mathbf{K}(E))$ such that

$$
\sigma(y)=\zeta_{2 n} y \quad \text { and } \quad \operatorname{div}_{E}\left(y^{2 n}\right)=(n+1) P_{1}^{\prime}+(n-1) P_{2}^{\prime}-2 n Q^{\prime} .
$$

Since $n$ is even, we get $(2 n, n+1)=(2 n, n-1)=1$. Therefore the branch points of $\pi$ are $P_{1}^{\prime}$ and $P_{2}^{\prime}$ whose ramification indices are $2 n$. Therefore

$$
\operatorname{div}(y)=(n+1) P_{1}+(n-1) P_{2}-\pi^{*}\left(Q^{\prime}\right) .
$$

Moreover, by the Riemann-Hurwitz formula we have $g(C)=2 n=g$. Hence

$$
\operatorname{div}(d y)=n P_{1}+(n-2) P_{2}-2 \pi^{*}\left(Q^{\prime}\right)+\sum_{i=1}^{3} \pi^{*}\left(R_{i}^{\prime}\right),
$$

where $R_{i}^{\prime}$ 's are points of $E$ which are distinct from $P_{1}^{\prime}, P_{2}^{\prime}$ and $Q^{\prime}$.

Let $D_{0}^{\prime}$ and $D_{2 l}^{\prime}(1 \leq l \leq n-1)$ be as in Section 2. Moreover, we set

$$
D_{n-1}^{\prime}=D_{2(n / 2-1)+1}^{\prime}=-n Q^{\prime}+\left(\frac{n}{2}-1\right) P_{1}^{\prime}+\left(\frac{n}{2}-1\right) P_{2}^{\prime}+\sum_{i=1}^{3} R_{i}^{\prime}
$$


and

$$
D_{n+1}^{\prime}=D_{2 \cdot n / 2+1}^{\prime}=-(n+2) Q^{\prime}+\left(\frac{n}{2}+1\right) P_{1}^{\prime}+\left(\frac{n}{2}-1\right) P_{2}^{\prime}+\sum_{i=1}^{3} R_{i}^{\prime} .
$$

Then $D_{0}^{\prime} \sim 0$. Moreover, for any $l$ with $1 \leq l \leq n-1$ we have $l\left(D_{2 l}^{\prime}\right)=1$ and $l\left(D_{2 l}^{\prime}-P_{1}^{\prime}\right)=l\left(D_{2 l}^{\prime}-P_{2}^{\prime}\right)=0$. In fact, first assume $l\left(D_{2 l}^{\prime}-P_{1}^{\prime}\right)=1$. Then $0 \sim D_{2 l}^{\prime}-P_{1}^{\prime}-D_{0}^{\prime} \sim 4 l P_{1}^{\prime}-4 l Q^{\prime}$, which implies that $2 n-1$ divides $4 l$. In view of $1 \leq l \leq n-1$ we must have $4 l=2 n-1$, which is a contradiction. Secondly, assume $l\left(D_{2 l}^{\prime}-P_{2}^{\prime}\right)=1$. Then $0 \sim D_{2 l}^{\prime}-P_{2}^{\prime}-D_{0}^{\prime} \sim-(4 l-2) Q^{\prime}+(4 l-2) P_{1}^{\prime}$, which implies that $2 n-1$ divides $4 l-2$. This is a contradiction. Now we have

$$
D_{n-1}^{\prime}-P_{1}^{\prime}-D_{0}^{\prime} \sim(2 n-1) P_{1}^{\prime}-(2 n-1) Q^{\prime} \sim 0,
$$

which implies that $l\left(D_{n-1}^{\prime}\right)=l\left(D_{n-1}^{\prime}-P_{1}^{\prime}\right)=1$ and $l\left(D_{n-1}^{\prime}-2 P_{1}^{\prime}\right)=0$. Moreover, $D_{n+1}^{\prime}-P_{2}^{\prime}-D_{0}^{\prime} \sim-(2 n-1) Q^{\prime}+(2 n-1) P_{1}^{\prime} \sim 0$, which implies that $l\left(D_{n+1}^{\prime}\right)=l\left(D_{n+1}^{\prime}-P_{2}^{\prime}\right)=1$ and $l\left(D_{n+1}^{\prime}-2 P_{2}^{\prime}\right)=0$.

Let $f \in \mathbf{K}(E)$ and set

$$
\operatorname{div}_{E}(f)=\sum_{P^{\prime} \in E} m\left(P^{\prime}\right) P^{\prime} .
$$

Then for any non-negative integer $r$ we obtain

$$
\begin{aligned}
\operatorname{div}_{C}\left(\frac{f d y}{y^{1-r}}\right)= & \left(2 n m\left(P_{1}^{\prime}\right)+n+(n+1)(r-1)\right) P_{1} \\
& +\left(2 n m\left(P_{2}^{\prime}\right)+n-2+(n-1)(r-1)\right) P_{2} \\
& +\left(m\left(Q^{\prime}\right)-r-1\right) \pi^{*}\left(Q^{\prime}\right) \\
& +\sum_{i=1}^{3}\left(m\left(R_{i}^{\prime}\right)+1\right) \pi^{*}\left(R_{i}^{\prime}\right)+\sum_{P^{\prime} \in S} m\left(P^{\prime}\right) \pi^{*}\left(P^{\prime}\right),
\end{aligned}
$$

where we set $S=E \backslash\left\{P_{1}^{\prime}, P_{2}^{\prime}, Q^{\prime}, R_{1}^{\prime}, R_{2}^{\prime}, R_{3}^{\prime}\right\}$.

For each $r \in\{0,2, \ldots, 2 n-2\} \cup\{n-1\} \cup\{n+1\}$ we take a nonzero element $f_{r} \in L\left(D_{r}^{\prime}\right)$ and set $\phi_{r}=f_{r} d y / y^{1-r}$. Then, by the above, $\operatorname{ord}_{P_{i}}\left(\phi_{0}\right)=2 n-1=g-1$ for $i=1,2$. For any $l$ with $1 \leq l \leq n-1$ we have $\operatorname{ord}_{P_{1}}\left(\phi_{2 l}\right)=2 l-1$ and $\operatorname{ord}_{P_{2}}\left(\phi_{2 l}\right)=2(n-l)-1$. Moreover,

$$
\begin{gathered}
\operatorname{ord}_{P_{1}}\left(\phi_{n-1}\right)=4 n-1-1=2 g-1-1, \\
\operatorname{ord}_{P_{2}}\left(\phi_{n-1}\right) \geq-2 n\left(\frac{n}{2}-1\right)+n-2+(n-1)(n-2)=0,
\end{gathered}
$$

$\operatorname{ord}_{P_{1}}\left(\phi_{n+1}\right) \geq-2 n\left(\frac{n}{2}+1\right)+n+(n+1) n=0$ and $\operatorname{ord}_{P_{2}}\left(\phi_{n+1}\right)=2 g-1-1$.

Hence $\phi_{0}, \phi_{2}, \ldots, \phi_{2 n-2}, \phi_{n-1}, \phi_{n+1}$ are regular 1-forms on $C$. Therefore we get $G\left(P_{i}\right) \supset\{2,4, \ldots, g-2, g, 2 g-1\}$ for $i=1,2$. 
Now let $C_{0}$ be the curve whose function field $\mathbf{K}\left(C_{0}\right)$ is $\mathbf{K}(E)\left(z^{1 / n}\right)$. Moreover, $\eta: C_{0} \rightarrow E$ denotes the surjective morphism of curves corresponding to the inclusion $\mathbf{K}(E) \subset \mathbf{K}\left(C_{0}\right)$. Let $\pi_{0}: C \rightarrow C_{0}$ be the double covering corresponding to the inclusion $\mathbf{K}\left(C_{0}\right) \subset \mathbf{K}(C)$. Since $\pi=\eta \circ \pi_{0}: C \rightarrow E$ has only two ramification points $P_{1}$ and $P_{2}$, which are totally ramified, by the Riemann-Hurwitz formula we get $g\left(C_{0}\right)=g / 2$. Moreover, $P_{1}$ and $P_{2}$ are ramification points of $\pi_{0}$. Therefore by Proposition 3 we obtain $G\left(P_{1}\right)=G\left(P_{2}\right)=\{1,2, \ldots, g-2, g, 2 g-1\}$.

5. The case $g \equiv 2 \bmod 4$. First we show the following arithmetic lemma which is the key to proving the next Proposition 5.

Key Lemma 4. Let $l \geq 2$ be an integer and let $p_{1}, \ldots, p_{l}$ be distinct prime numbers. Then there is a partition

$$
\left\{i_{1}, \ldots, i_{t}\right\} \cup\left\{i_{t+1}, \ldots, i_{l}\right\}=\{1, \ldots, l\}
$$

with $1 \leq t \leq l-1$ such that $\left(4 p_{i_{1}} \ldots p_{i_{t}}+1, p_{i_{t+1}} \ldots p_{i_{l}}\right)=1$.

Proof. We may assume that $p_{1}, \ldots, p_{l}$ are odd. In fact, if $p_{1}=2$, then $\left(4 p_{2} \ldots p_{l}+1, p_{1}\right)=1$. We prove the lemma by induction on $l \geq 2$.

Let $l=2$. We may assume that $p_{1}<p_{2}$. Suppose that

$$
\left(4 p_{1}+1, p_{2}\right) \neq 1 \text { and }\left(4 p_{2}+1, p_{1}\right) \neq 1,
$$

which implies that $p_{2} \mid\left(4 p_{1}+1\right)$ and $p_{1} \mid\left(4 p_{2}+1\right)$. Let $4 p_{1}+1=m p_{2}$. Then $m$ must be 1 or 3 . Moreover, $p_{1}$ divides $\left(4 p_{2}+1\right) m=16 p_{1}+4+m$, which implies that $p_{1} \mid(4+m)$. Let $m=1$. Then $p_{1} \mid 5$, which implies that $p_{1}=5$. Hence $p_{2}=4 p_{1}+1=21$ is not prime, a contradiction. Let $m=3$. Then $p_{1} \mid 7$, which implies that $p_{1}=7$. Hence $3 p_{2}=4 p_{1}+1=29$, a contradiction.

Let $l \geq 3$. We may assume that $p_{l}>p_{j}$ for all $j \neq l$. Suppose that

$$
\left(4 p_{1} \ldots p_{i-1} p_{i+1} \ldots p_{l}+1, p_{i}\right) \neq 1, \quad \text { i.e., } \quad p_{i} \mid\left(4 p_{1} \ldots p_{i-1} p_{i+1} \ldots p_{l}+1\right)
$$

for all $i=1, \ldots, l$. Then $p_{l} \nmid\left(4 p_{1} \ldots p_{i-1} p_{i+1} \ldots p_{l-1}+1\right)$ for all $i=1, \ldots$, $l-1$. In fact, suppose that $p_{l} \mid\left(4 p_{1} \ldots p_{i-1} p_{i+1} \ldots p_{l-1}+1\right)$ for some $i$. In view of $p_{l} \mid\left(4 p_{1} \ldots p_{l-1}+1\right)$ we get

$$
p_{l} \mid 4 p_{1} \ldots p_{i-1} p_{i+1} \ldots p_{l-1}\left(p_{i}-1\right),
$$

which implies that $p_{l} \mid\left(p_{i}-1\right)$. This contradicts $p_{l}>p_{j}$ for all $j \neq l$.

Moreover, we may assume that $p_{i} \mid\left(4 p_{1} \ldots p_{i-1} p_{i+1} \ldots p_{l-1}+1\right)$ for each $i=1, \ldots, l-1$. In fact, suppose that $p_{i} \nmid\left(4 p_{1} \ldots p_{i-1} p_{i+1} \ldots p_{l-1}+1\right)$ for some $i$. In view of $p_{l} \nmid\left(4 p_{1} \ldots p_{i-1} p_{i+1} \ldots p_{l-1}+1\right)$ we obtain a partition

$$
\{1, \ldots, i-1, i+1, \ldots, l-1\} \cup\{i, l\}=\{1, \ldots, l\}
$$

such that $\left(p_{i} p_{l}, 4 p_{1} \ldots p_{i-1} p_{i+1} \ldots p_{l-1}+1\right)=1$. Hence

$$
p_{i} \mid 4 p_{1} \ldots p_{i-1} p_{i+1} \ldots p_{l-1}\left(p_{l}-1\right)
$$


for each $i=1, \ldots, l-1$. Therefore $p_{i} \mid\left(p_{l}-1\right)$ for all $i=1, \ldots, l-1$, which implies that $p_{l}-1=m p_{1} \ldots p_{l-1}$ for some integer $m$. If $m \geq 5$, then $p_{l} \geq 5 p_{1} \ldots p_{l-1}+1$, which contradicts $p_{l} \mid\left(4 p_{1} \ldots p_{l-1}+1\right)$. If $m \leq 3$, then $\left(m p_{1} \ldots p_{l-1}+1\right) \mid\left(4 p_{1} \ldots p_{l-1}+1\right)$, a contradiction.

Hence $m=4$. By the induction hypothesis there is a partition

$$
\left\{i_{1}, \ldots, i_{t}\right\} \cup\left\{i_{t+1}, \ldots, i_{l-1}\right\}=\{1, \ldots, l-1\}
$$

with $1 \leq t \leq l-2$ such that $\left(4 p_{i_{1}} \ldots p_{i_{t}}+1, p_{i_{t+1}} \ldots p_{i_{l-1}}\right)=1$. In view of $p_{l}=4 p_{1} \ldots p_{l-1}+1>4 p_{i_{1}} \ldots p_{i_{t}}+1$ we get $p_{l} \nmid\left(4 p_{i_{1}} \ldots p_{i_{t}}+1\right)$. Hence we obtain $\left(4 p_{i_{1}} \ldots p_{i_{t}}+1, p_{i_{t+1}} \ldots p_{i_{l-1}} p_{l}\right)=1$.

Using the Key Lemma we show the following proposition, which is crucial to the proof of the remaining case of the Main Theorem.

Proposition 5. Let $n=10 t+3$ with an integer $t \geq 1$. Then there exists an integer $s$ with $3 \leq s \leq(n-3) / 2$ such that $s \mid(2 n-1)$ and $(2 n-1, n+2 s)$ $=1$.

Proof. First, we consider the case $2 n-1=p_{1}^{e} p_{2} \ldots p_{r}$ with $e \geq 2$ if $p_{1} \geq 5$ or $e \geq 3$ if $p_{1}=3$, where $p_{2}, \ldots, p_{r}$ may not be distinct. Let $s=p_{1} p_{2} \ldots p_{r}$ and $q=p_{1}^{e-1}$. Then $s \mid(2 n-1)$ and

$$
\begin{aligned}
(2 n-1, n+2 s) & =(2 n-1,2 n+4 s)=(2 n-1,4 s+1) \\
& =(s q, 4 s+1)=(q, 4 s+1)=\left(p_{1}^{e-1}, 4 p_{1} p_{2} \ldots p_{r}+1\right)=1 .
\end{aligned}
$$

Moreover,

$$
s=p_{1} p_{2} \ldots p_{r}=\frac{2 n-1}{q} \leq \frac{2 n-1}{5} \leq \frac{n-3}{2}
$$

because $q=p_{1}^{e-1} \geq 5$ and $n \geq 13$.

Secondly, we consider the case $2 n-1=p_{1}^{2} p_{2} \ldots p_{r}$ with $p_{1}=3$ where $p_{1}, \ldots, p_{r}$ are distinct. In view of $2 n-1=5(4 t+1)$ we have $r \geq 2$. By Lemma 4 we have a partition

$$
\left\{i_{1}, \ldots, i_{t}\right\} \cup\left\{i_{t+1}, \ldots, i_{r}\right\}=\{1, \ldots, r\}
$$

with $1 \leq t \leq r-1$ such that $\left(4 p_{i_{1}} \ldots p_{i_{t}}+1, p_{i_{t+1}} \ldots p_{i_{r}}\right)=1$. Hence we get $\left(4 p_{i_{1}} \ldots p_{i_{t}}+1, p_{1} p_{i_{t+1}} \ldots p_{i_{r}}\right)=1$. Let $s=p_{i_{1}} \ldots p_{i_{t}}$ and $q=p_{1} p_{i_{t+1}} \ldots p_{i_{r}}$. Then $s \mid(2 n-1)$ and

$$
(2 n-1, n+2 s)=(q, 4 s+1)=\left(p_{1} p_{i_{t+1}} \ldots p_{i_{r}}, 4 p_{i_{1}} \ldots p_{i_{t}}+1\right)=1 .
$$

Moreover,

$$
s=\frac{2 n-1}{q} \leq \frac{2 n-1}{9}<\frac{n-3}{2}
$$

because $q=p_{1} p_{i_{t+1}} \ldots p_{i_{r}} \geq 9$. 
Lastly, we consider the case $2 n-1=p_{1} p_{2} \ldots p_{r}$ where $p_{1}, \ldots, p_{r}$ are distinct. By Lemma 4 we have a partition $\left\{i_{1}, \ldots, i_{t}\right\} \cup\left\{i_{t+1}, \ldots, i_{r}\right\}=$ $\{1, \ldots, r\}$ with $1 \leq t \leq r-1$ such that $\left(4 p_{i_{1}} \ldots p_{i_{t}}+1, p_{i_{t+1}} \ldots p_{i_{r}}\right)=1$.

Let $t \leq r-2$ or $p_{i}>3$ for all $i$. We set $s=p_{i_{1}} \ldots p_{i_{t}}$ and $q=p_{i_{t+1}} \ldots p_{i_{r}}$. Then $s \mid(2 n-1)$ and $(2 n-1, n+2 s)=1$. Moreover,

$$
s=\frac{2 n-1}{q}=\frac{2 n-1}{p_{i_{t+1}} \ldots p_{i_{r}}} \leq \frac{2 n-1}{5} \leq \frac{n-3}{2}
$$

because $n \geq 13$.

Let $t=r-1$ and $p_{i}=3$ for some $i$. In this case $r \geq 3$, because $2 n-1=$ $5(4 t+1)$ with $4 t+1 \geq 5$. Then we may assume that $p_{1}=3$. Let $p_{r}>p_{j}$ for all $j \neq r$. Moreover, we may assume either

(1) $\left(p_{i}, 4 p_{1} \ldots p_{i-1} p_{i+1} \ldots p_{r}+1\right)=1$ for some $i=2, \ldots, r$, or

(2) there exists a partition

$$
\left\{i_{1}, \ldots, i_{t}\right\} \cup\left\{i_{t+1}, \ldots, i_{r-1}\right\}=\{1, \ldots, r-1\}
$$

with $1 \leq t \leq r-2$ such that $\left(p_{i_{t+1}} \ldots p_{i_{r-1}} p_{r}, 4 p_{i_{1}} \ldots p_{i_{t}}+1\right)=1$.

In fact, suppose that (1) does not hold, i.e.,

$$
p_{i} \mid\left(4 p_{1} \ldots p_{i-1} p_{i+1} \ldots p_{r}+1\right) \quad \text { for all } i=2, \ldots, r .
$$

Then

$$
p_{r} \nmid\left(4 p_{1} \ldots p_{i-1} p_{i+1} \ldots p_{r-1}+1\right) \quad \text { for all } i=2, \ldots, r-1 .
$$

In fact, suppose that

$$
p_{r} \mid\left(4 p_{1} \ldots p_{i-1} p_{i+1} \ldots p_{r-1}+1\right) \quad \text { for some } i=2, \ldots, r-1 .
$$

In view of $p_{r} \mid\left(4 p_{1} \ldots p_{r-1}+1\right)$ we obtain $p_{r} \mid 4 p_{1} \ldots p_{i-1} p_{i+1} \ldots p_{r-1}\left(p_{i}-1\right)$, which implies that $p_{r} \mid\left(p_{i}-1\right)$. This contradicts $p_{r}>p_{i}$.

Moreover, we may assume that

$$
p_{i} \mid\left(4 p_{1} \ldots p_{i-1} p_{i+1} \ldots p_{r-1}+1\right) \quad \text { for all } i=2, \ldots, r-1 .
$$

In fact, suppose that

$$
p_{i} \nmid\left(4 p_{1} \ldots p_{i-1} p_{i+1} \ldots p_{r-1}+1\right) \quad \text { for some } i=2, \ldots, r-1 .
$$

In view of $p_{r} \nmid\left(4 p_{1} \ldots p_{i-1} p_{i+1} \ldots p_{r-1}+1\right)$ we have a partition

$$
\{1, \ldots, i-1, i+1, \ldots, r-1\} \cup\{i, r\}=\{1, \ldots, r\}
$$

such that $\left(p_{i} p_{r}, 4 p_{1} \ldots p_{i-1} p_{i+1} \ldots p_{r-1}+1\right)=1$. This case reduces to the case $t \leq r-2$ in which we have already proven the statement. Hence in view of

$$
p_{i} \mid\left(4 p_{1} \ldots p_{i-1} p_{i+1} \ldots p_{r}+1\right) \quad \text { for all } i=2, \ldots, r-1
$$

we have $p_{i} \mid 4 p_{1} \ldots p_{i-1} p_{i+1} \ldots p_{r-1}\left(p_{r}-1\right)$ for all $i=1, \ldots, r-1$, which implies $p_{i} \mid\left(p_{r}-1\right)$ for all $i=2, \ldots, r-1$. Therefore $p_{2} \ldots p_{r-1} \mid\left(p_{r}-1\right)$, 
which in turn implies that $p_{r}-1=m p_{2} \ldots p_{r-1}$ where $m$ is even. In view of $p_{r} \mid\left(4 p_{1} p_{2} \ldots p_{r-1}+1\right)$ with $p_{1}=3$ we have

$$
12 p_{2} \ldots p_{r-1}+1=m^{\prime} p_{r}=m^{\prime}\left(m p_{2} \ldots p_{r-1}+1\right)=m^{\prime} m p_{2} \ldots p_{r-1}+m^{\prime}
$$

with a positive integer $m^{\prime}$. Then we must have $m^{\prime}=1$, i.e., $m=12$. In fact, suppose that $m^{\prime} \geq 2$. Then $12-m^{\prime} m>0$, which implies that $12>m^{\prime} m \geq$ $2 m^{\prime}$. Hence $m^{\prime} \leq 5$, which implies that

$$
4 \geq m^{\prime}-1=\left(12-m^{\prime} m\right) p_{2} \ldots p_{r-1} \geq p_{2} \ldots p_{r-1} \geq 5 p_{3} \ldots p_{r-1} .
$$

This is a contradiction. Hence $m^{\prime}=1$.

Therefore we obtain

$$
p_{r}=12 p_{2} \ldots p_{r-1}+1=4 p_{1} p_{2} \ldots p_{r-1}+1 .
$$

Since $p_{1}, p_{2}, \ldots, p_{r-1}$ are distinct primes and $r-1 \geq 2$, by Lemma 4 there exists a partition $\left\{i_{1}, \ldots, i_{t}\right\} \cup\left\{i_{t+1}, \ldots, i_{r-1}\right\}=\{1, \ldots, r-1\}$ with $1 \leq t \leq r-2$ such that $\left(4 p_{i_{1}} \ldots p_{i_{t}}+1, p_{i_{t+1}} \ldots p_{i_{r-1}}\right)=1$. In view of $p_{r}=4 p_{1} p_{2} \ldots p_{r-1}+1>4 p_{i_{1}} \ldots p_{i_{t}}+1$ we have $p_{r} \nmid\left(4 p_{i_{1}} \ldots p_{i_{t}}+1\right)$. Hence $\left(4 p_{i_{1}} \ldots p_{i_{t}}+1, p_{i_{t+1}} \ldots p_{i_{r-1}} p_{r}\right)=1$. Thus we have proven that if $t=r-1$ and $p_{1}=3$, then we may assume that either (1) or (2) holds.

In case (1) (resp. (2)) we set $s=p_{1} \ldots p_{i-1} p_{i+1} \ldots p_{r}\left(\right.$ resp. $\left.s=p_{i_{1}} \ldots p_{i_{t}}\right)$ and $q=p_{i} \geq 5$ (resp. $\left.q=p_{i_{t+1}} \ldots p_{i_{r-1}} p_{r} \geq 15\right)$. Then we have $s \mid(2 n-1)$ and $(2 n-1, n+2 s)=(q, 4 s+1)=1$. Moreover,

$$
s=\frac{2 n-1}{q} \leq \frac{2 n-1}{5} \leq \frac{n-3}{2}
$$

because $n \geq 13$.

Now we prove the Main Theorem in the case $g \equiv 2 \bmod 4$ with $g \geq 10$.

Let $g=2 n$ where $n$ is an odd integer $\geq 5$. First we show that there exists an odd integer $s$ with $1 \leq s \leq(n-3) / 2$ such that

$$
s \mid(2 n-1) \text { and }(2 n-1, n+2 s)=1 .
$$

In fact, let $g \not \equiv 1 \bmod 5$, which implies that $n+2 \not \equiv 0 \bmod 5$. Then

$$
(2 n-1, n+2)=(2 n-1,2 n+4)=(2 n-1,5)=1 .
$$

Hence in this case we may take $s=1$. Let $g \equiv 1 \bmod 5$. Then we can write $n=10 t+3$ with $t \geq 1$. By Proposition 5 we may take an integer $s$ with $3 \leq s \leq(n-3) / 2$ such that $s \mid(2 n-1)$ and $(2 n-1, n+2 s)=1$.

Now there exists a unique integer $m$ with $0<m \leq 2 n-3$ such that

$$
(m+1)(n+2 s) \equiv 1 \bmod 2 n-1 .
$$

In fact, in view of $(2 n-1, n+2 s)=1$ there exists a unique integer $0 \leq$ $m \leq 2 n-3$ such that $(m+1)(n+2 s) \equiv 1 \bmod 2 n-1$. If $m=0$, then 
$n+2 s \equiv 1 \bmod 2 n-1$. Since

$$
n+2 s-1 \geq n+1>0 \quad \text { and } \quad n+2 s-1 \leq n+2 \cdot \frac{n-3}{2}-1=2 n-4,
$$

this contradicts $(2 n-1) \mid(n+2 s-1)$.

Let $E$ be an elliptic curve over $k$ with the origin $Q^{\prime}$. Let $P_{1}^{\prime}$ be a point of $E$ such that $(2 n-1)\left[P_{1}^{\prime}\right]=\left[Q^{\prime}\right]$ and $h\left[P_{1}^{\prime}\right] \neq\left[Q^{\prime}\right]$ for any positive integer $h<2 n-1$. Moreover, $P_{2}^{\prime}$ denotes the point of $E$ such that $\left[P_{2}^{\prime}\right]=-m\left[P_{1}^{\prime}\right]$, i.e., $P_{2}^{\prime} \sim-m P_{1}^{\prime}+(m+1) Q^{\prime}$. Then $P_{1}^{\prime}, P_{2}^{\prime}$ and $Q^{\prime}$ are distinct because $0<m \leq 2 n-3$. Now we obtain

$$
(n-2 s) P_{1}^{\prime}+(n+2 s) P_{2}^{\prime} \sim 2 n Q^{\prime} .
$$

In fact,

$(n-2 s) P_{1}^{\prime}+(n+2 s) P_{2}^{\prime} \sim(-m(n+2 s)+n-2 s) P_{1}^{\prime}+(n+2 s)(m+1) Q^{\prime}$.

Then $-m(n+2 s)+n-2 s \equiv-1+2 n \equiv 0 \bmod 2 n-1$ because $(m+1)(n+2 s) \equiv$ $1 \bmod 2 n-1$. Hence

$$
\begin{aligned}
& (n-2 s) P_{1}^{\prime}+(n+2 s) P_{2}^{\prime} \\
& \quad \sim \frac{-m(n+2 s)+n-2 s}{2 n-1}(2 n-1) P_{1}^{\prime}+(n+2 s)(m+1) Q^{\prime} \sim 2 n Q^{\prime} .
\end{aligned}
$$

Hence we may take $z \in \mathbf{K}(E)$ such that

$$
\operatorname{div}(z)=(n-2 s) P_{1}^{\prime}+(n+2 s) P_{2}^{\prime}-2 n Q^{\prime} .
$$

Let $C$ be the curve whose function field $\mathbf{K}(C)$ is $\mathbf{K}(E)\left(z^{1 /(2 n)}\right)$. Moreover, $\pi: C \rightarrow E$ denotes the surjective morphism of curves corresponding to the inclusion $\mathbf{K}(E) \subset \mathbf{K}(C)$. Then we may take $y \in \mathbf{K}(C)$ and $\sigma \in \operatorname{Aut}(\mathbf{K}(C) / \mathbf{K}(E))$ such that

$$
\sigma(y)=\zeta_{2 n} y \quad \text { and } \quad \operatorname{div}_{E}\left(y^{2 n}\right)=(n-2 s) P_{1}^{\prime}+(n+2 s) P_{2}^{\prime}-2 n Q^{\prime} .
$$

Now we have $(n, s)=1$. In fact, $(n, s) \mid(2 n-1, n+2 s)$ because $s \mid(2 n-1)$, which implies that $(n, s)=1$. Therefore $(2 n, n+2 s)=(s, n)=1$ and $(2 n, n-2 s)=1$, because $n$ is odd. Therefore the branch points of $\pi$ are $P_{1}^{\prime}$ and $P_{2}^{\prime}$ whose ramification indices are $2 n$. Thus

$$
\operatorname{div}(y)=(n-2 s) P_{1}+(n+2 s) P_{2}-\pi^{*}\left(Q^{\prime}\right) .
$$

Moreover, by the Riemann-Hurwitz formula we have $g(C)=2 n=g$. Hence

$$
\operatorname{div}(d y)=(n-2 s-1) P_{1}+(n+2 s-1) P_{2}-2 \pi^{*}\left(Q^{\prime}\right)+\sum_{i=1}^{3} \pi^{*}\left(R_{i}^{\prime}\right),
$$

where $R_{i}^{\prime}$ 's are points of $E$ which are distinct from $P_{1}^{\prime}, P_{2}^{\prime}$ and $Q^{\prime}$.

We set

$$
D_{0}^{\prime}=-P_{1}^{\prime}-P_{2}^{\prime}-Q^{\prime}+\sum_{i=1}^{3} R_{i}^{\prime}
$$


which is linearly equivalent to zero. Let $l \in\{0,1, \ldots, 2 s-1\}$ be fixed. Then for any even $r>0$ with

$$
\frac{2 l n-1}{2 s}<r \leq \frac{2(l+1) n-1}{2 s}
$$

we set

$$
D_{r}^{\prime}=-(r+1) Q^{\prime}+\left(\frac{r}{2}-l-1\right) P_{1}^{\prime}+\left(\frac{r}{2}+l\right) P_{2}^{\prime}+\sum_{i=1}^{3} R_{i}^{\prime} .
$$

Next we show that for any $r, l\left(D_{r}^{\prime}-P_{1}^{\prime}\right)=0$ and $l\left(D_{r}^{\prime}-P_{2}^{\prime}\right)=0$, i.e., $D_{r}^{\prime}-P_{1}^{\prime} \nsim 0$ and $D_{r}^{\prime}-P_{2}^{\prime} \nsim 0$. Suppose that $D_{r}^{\prime}-P_{1}^{\prime} \sim 0$. Then $0 \sim D_{r}^{\prime}-P_{1}^{\prime}-D_{0}^{\prime}$, which implies that

$$
\left(\left(\frac{r}{2}+l+1\right)(m+1)-r\right) Q^{\prime} \sim\left(\left(\frac{r}{2}+l+1\right)(m+1)-r\right) P_{1}^{\prime} .
$$

Hence

$$
\left(\frac{r}{2}+l+1\right)(m+1)-r \equiv 0 \bmod 2 n-1 .
$$

In view of $s \mid(2 n-1)$, we get

$$
\left(\frac{r}{2}+l+1\right)(m+1)-r \equiv 0 \bmod s .
$$

Moreover, since $(m+1)(n+2 s) \equiv 1 \bmod 2 n-1$ we have $(m+1) n \equiv 1 \bmod s$. Hence

$$
0 \equiv 2\left(\frac{r}{2}+l+1\right)(m+1) n-2 r n \equiv 2(l+1) \bmod s,
$$

which implies that $l+1 \equiv 0 \bmod s$. In view of $0 \leq l \leq 2 s-1$ we have $l=s-1$ or $2 s-1$.

Let $l=s-1$. Then $r$ satisfies

$$
\frac{2(s-1) n-1}{2 s}<r \leq \frac{2 s n-1}{2 s} .
$$

Moreover,

$$
\left(\frac{r}{2}+s\right)(m+1) \equiv r \bmod 2 n-1 .
$$

In view of $(m+1)(n+2 s) \equiv 1 \bmod 2 n-1$ we have

$$
\begin{aligned}
\frac{r}{2}+s & \equiv\left(\frac{r}{2}+s\right)(m+1)(n+2 s) \equiv r(n+2 s) \\
& \equiv \frac{r}{2}(1+4 s) \bmod 2 n-1,
\end{aligned}
$$

which implies that $s(2 r-1) \equiv 0 \bmod 2 n-1$. Hence we may set

$$
2 r-1=\frac{2 n-1}{s} \cdot k \quad \text { with a positive odd integer } k .
$$


Then

$$
\frac{2(s-1) n-1}{2 s}<r=\frac{(2 n-1) k+s}{2 s} \leq \frac{2 s n-1}{2 s},
$$

which implies that $2(k-s) n \leq k-s-1<2(k-s+1) n$. If $k>s$, then

$$
2 n \leq \frac{k-s-1}{k-s}=1-\frac{1}{k-s}<1,
$$

a contradiction. If $k=s$, then $0 \leq-1$, a contradiction. Let $k-s=-1$. Since $k$ and $s$ are odd, this is a contradiction. If $k-s<-1$, then

$$
2 n<\frac{k-s-1}{k-s+1}=1+\frac{2}{-k+s-1} \leq 3,
$$

a contradiction.

Let $l=2 s-1$. Then $r$ satisfies

$$
\frac{2(2 s-1) n-1}{2 s}<r \leq \frac{4 s n-1}{2 s} .
$$

Moreover,

$$
\left(\frac{r}{2}+2 s\right)(m+1) \equiv r \bmod 2 n-1 .
$$

Hence

$$
\frac{r}{2}+2 s \equiv\left(\frac{r}{2}+2 s\right)(m+1)(n+2 s) \equiv \frac{r}{2}(1+4 s) \bmod 2 n-1,
$$

which implies that $2 s(r-1) \equiv 0 \bmod 2 n-1$. Therefore we may set

$$
r-1=\frac{2 n-1}{s} \cdot k \quad \text { with a positive odd integer } k .
$$

Then

$$
\frac{2(2 s-1) n-1}{2 s}<r=\frac{(4 n-2) k+2 s}{2 s} \leq \frac{4 s n-1}{2 s},
$$

which implies that $4(k-s) n \leq 2 k-2 s-1<2(2 k-2 s+1) n$. This is a contradiction.

Moreover, we prove that $D_{r}^{\prime}-P_{2}^{\prime} \nsim 0$. Suppose that $D_{r}^{\prime}-P_{2}^{\prime} \sim 0$. Then $0 \sim D_{r}^{\prime}-P_{2}^{\prime}-D_{0}^{\prime}$, which implies that

$$
\left(\left(\frac{r}{2}+l\right)(m+1)-r\right) Q^{\prime} \sim\left(\left(\frac{r}{2}+l\right)(m+1)-r\right) P_{1}^{\prime} .
$$

Hence

$$
\left(\frac{r}{2}+l\right)(m+1)-r \equiv 0 \bmod 2 n-1
$$

In view of $s \mid(2 n-1)$, we get

$$
\left(\frac{r}{2}+l\right)(m+1)-r \equiv 0 \bmod s
$$


Since $(m+1) n \equiv 1 \bmod s$, we obtain

$$
0 \equiv\left(\frac{r}{2}+l\right)(m+1) n-r n \equiv r / 2+l-n r \bmod s,
$$

which implies that $0 \equiv r+2 l-2 n r \equiv 2 l \bmod s$. Since $s$ is odd, we have $l \equiv 0 \bmod s$, which implies that $l=0$ or $l=s$.

Let $l=0$. Then $2 \leq r \leq(2 n-1) /(2 s)$. Moreover,

$$
\frac{r}{2}(m+1) \equiv r \bmod 2 n-1 \text {. }
$$

Hence

$$
\frac{r}{2} \equiv \frac{r}{2}(m+1)(n+2 s) \equiv 2 s r+\frac{r}{2} \bmod 2 n-1,
$$

which implies that $0 \equiv 2 s r \bmod 2 n-1$. Therefore $r \equiv 0 \bmod (2 n-1) / s$, which contradicts $2 \leq r \leq(2 n-1) /(2 s)$.

Let $l=s$. Then

$$
\frac{2 s n-1}{2 s}<r \leq \frac{2(s+1) n-1}{2 s} .
$$

Moreover,

$$
\left(\frac{r}{2}+s\right)(m+1) \equiv r \bmod 2 n-1 .
$$

Hence

$$
\frac{r}{2}+s \equiv\left(\frac{r}{2}+s\right)(m+1)(n+2 s) \equiv \frac{r}{2}(4 s+1) \bmod 2 n-1,
$$

which implies that $s \equiv 2 s r \bmod 2 n-1$. Hence we may set

$$
2 r-1=\frac{2 n-1}{s} \cdot k,
$$

where $k$ is an odd positive integer. If $k \geq s+2$, then

$$
\begin{aligned}
2 r-1 & \geq \frac{2 n-1}{s}(s+2)>2 n-1+\frac{2 n-1}{s} \\
& =\frac{2(s+1) n-1}{s}-1=2 \cdot \frac{2(s+1) n-1}{2 s}-1 \geq 2 r-1,
\end{aligned}
$$

a contradiction. Now we have

$$
2 r-1>2 \cdot \frac{2 s n-1}{2 s}-1=2 n-\frac{1}{s}-1,
$$

which implies that $2 r-1 \geq 2 n-1$. If $k \leq s-2$, then

$$
2 n-1 \leq 2 r-1 \leq \frac{2 n-1}{s}(s-2)=2 n-1-\frac{2(2 n-1)}{s}<2 n-1,
$$


a contradiction. Hence $k=s$, which implies that

$$
2 r-1=\frac{2 n-1}{s} \cdot s=2 n-1 .
$$

Therefore $r=n$. Since $r$ is even and $n$ is odd, this is a contradiction. Hence $D_{r}^{\prime}-P_{2}^{\prime} \nsim 0$. Thus we obtain the following: Let $l \in\{0,1, \ldots, 2 s-1\}$ be fixed. Then for any even $r>0$ with $(2 l n-1) /(2 s)<r \leq(2(l+1) n-1) /(2 s)$ we get

$$
l\left(D_{r}^{\prime}\right)=1 \quad \text { and } \quad l\left(D_{r}^{\prime}-P_{1}^{\prime}\right)=l\left(D_{r}^{\prime}-P_{2}^{\prime}\right)=0 .
$$

Now in view of $(n, s)=1$ there is a unique non-negative integer $q \leq 2 s-1$ such that $(2 q+1) n \equiv 2 s+1 \bmod 4 s$. Then we set

$$
\begin{aligned}
r_{1} & =2 \cdot \frac{2 s+1-(2 q+1) n+4 s(n-1)}{4 s}+1 \\
& =2 \cdot \frac{(4 s-2 q-1) n-(2 s-1)}{4 s}+1 .
\end{aligned}
$$

Note that $r_{1}$ is an odd integer $\geq 3$. In fact,

$$
4 s-2 q-1 \geq 4 s-2(2 s-1)-1=1 .
$$

Hence in view of $s \leq(n-3) / 2$ we get

$$
(4 s-2 q-1) n-(2 s-1) \geq n-(2 s-1) \geq 2 s+3-(2 s-1)=4>0,
$$

which implies that $r_{1} \geq 3$. Then we define

$$
\begin{aligned}
D_{r_{1}}^{\prime}= & -\left(r_{1}+1\right) Q^{\prime}+\frac{(4 s-2 q-1) n-(2 s-1)-4 s(2 s-q)}{4 s} P_{1}^{\prime} \\
& +\frac{(4 s-2 q-1) n-(2 s-1)+4 s(2 s-q)}{4 s} P_{2}^{\prime}+\sum_{i=1}^{3} R_{i}^{\prime} .
\end{aligned}
$$

Note that $\operatorname{deg} D_{r_{1}}^{\prime}=1$. We prove that $D_{r_{1}}^{\prime}-P_{1}^{\prime} \sim 0$. In fact, in view of $P_{2}^{\prime} \sim(m+1) Q^{\prime}-m P_{1}^{\prime}$ we have

$$
\begin{aligned}
D_{r_{1}}^{\prime} & -P_{1}^{\prime}-D_{0}^{\prime} \\
& \sim \frac{(4 s-2 q-1) n(m-1)+(4 s(2 s-q)+2 s+1)(m+1)-2}{4 s}\left(Q^{\prime}-P_{1}^{\prime}\right) .
\end{aligned}
$$

Then

$$
\begin{aligned}
& (4 s-2 q-1) n(m-1)+(4 s(2 s-q)+2 s+1)(m+1)-2 \\
& =4 s(n(m-1)+2 s(m+1)) \\
& \quad-((2 q+1) n(m-1)-(2 s+1)(m-1)+4 s q(m+1)-4 s) .
\end{aligned}
$$

Let

$$
u=\frac{(n+2 s)(m+1)-1}{2 n-1}
$$


which is a positive integer because $(m+1)(n+2 s) \equiv 1 \bmod 2 n-1$. We have

$$
\begin{aligned}
&(2 q+1) n(m-1)-(2 s+1)(m-1)+4 s q(m+1)-4 s \\
&= 2 q((n+2 s)(m+1)-2 n)+(n+2 s)(m+1)-2 n-4 s m-m+1-4 s \\
&= 2 q((2 n-1) u+1-2 n)+(2 n-1) u+1-2 n \\
& \quad-2((n+2 s)(m+1)-n(m+1))-m+1 \\
&=(2 n-1)((2 q-1) u-2 q+m) .
\end{aligned}
$$

Now $(2 q-1) n=(2 q+1) n-2 n \equiv 2 s+1-2 n \equiv 0 \bmod s$, which implies that $s \mid(2 q-1)$ because $(n, s)=1$. Moreover,

$$
\begin{aligned}
(-2 q+m) n & =-2 q n+m n \equiv n-2 s-1+m n \\
& =(n+2 s)(m+1)-1-2 s-2 s m-2 s \equiv 0 \bmod s
\end{aligned}
$$

because $s \mid(2 n-1)$. In view of $(n, s)=1$ we get $s \mid(-2 q+m)$. Therefore $4 s \mid((2 q-1) u-2 q+m)$ because $(4,2 n-1)=1$, which implies that

$$
(2 n-1) 4 s \mid((2 q+1) n(m-1)-(2 s+1)(m-1)+4 s q(m+1)-4 s) .
$$

Moreover,

$$
4 s(n(m-1)+2 s(m+1))=4 s((m+1)(n+2 s)-1-(2 n-1)),
$$

which implies that $4 s(2 n-1) \mid 4 s(n(m-1)+2 s(m+1))$. Hence the integer

$$
\frac{(4 s-2 q-1) n(m-1)+(4 s(2 s-q)+2 s+1)(m+1)-2}{4 s}
$$

is divisible by $2 n-1$, which implies that $D_{r_{1}}^{\prime}-P_{1}^{\prime} \sim 0$.

Next we set

$$
r_{2}=\frac{(2 q+1) n-1}{2 s}=2 \cdot \frac{(2 q+1) n-(2 s+1)}{4 s}+1,
$$

which is an odd integer because $(2 q+1) n \equiv 2 s+1 \bmod 4 s$. Moreover, $3 \leq r_{2} \leq 2 n-3$. In fact, $1 \leq 2 q+1 \leq 4 s-1$ because $0 \leq q \leq 2 s-1$. Hence

$$
\frac{n-1}{2 s} \leq r_{2}=\frac{(2 q+1) n-1}{2 s} \leq \frac{(4 s-1) n-1}{2 s}=2 n-\frac{n+1}{2 s} .
$$

In view of $0<1 \leq s \leq(n-3) / 2$ we have

$$
1<\frac{n-1}{n-3} \leq \frac{n-1}{2 s} \text { and } 2 n-\frac{n+1}{2 s} \leq 2 n-\frac{n+1}{n-3}<2 n-1 .
$$

Now we set

$$
\begin{aligned}
D_{r_{2}}^{\prime}= & -\left(r_{2}+1\right) Q^{\prime}+\frac{(2 q+1) n-(2 s+1)-4 s q}{4 s} P_{1}^{\prime} \\
& +\frac{(2 q+1) n-(2 s+1)+4 s q}{4 s} P_{2}^{\prime}+\sum_{i=1}^{3} R_{i}^{\prime},
\end{aligned}
$$


which is of degree 1 . We prove that $D_{r_{2}}^{\prime}-P_{2}^{\prime} \sim 0$. We have $D_{r_{2}}^{\prime}-P_{2}^{\prime}-D_{0}^{\prime}$

$$
\sim \frac{(2 q+1) n(m-1)-(2 s+1)(m-1)+4 s q(m+1)-4 s}{4 s}\left(Q^{\prime}-P_{1}^{\prime}\right) .
$$

By the argument in the proof of $D_{r_{1}}^{\prime}-P_{1}^{\prime} \sim 0$ we show that

$$
\frac{(2 q+1) n(m-1)-(2 s+1)(m-1)+4 s q(m+1)-4 s}{4 s}
$$

is divisible by $2 n-1$, which implies that $D_{r_{2}}^{\prime}-P_{2}^{\prime} \sim D_{0}^{\prime} \sim 0$.

Now we are in a position to prove that $\{1, \ldots, g-2, g, 2 g-1\}$ is the gap sequence at $P_{1}$ and $P_{2}$. Let $f \in \mathbf{K}(E)$ and set

$$
\operatorname{div}_{E}(f)=\sum_{P^{\prime} \in E} m\left(P^{\prime}\right) P^{\prime}
$$

Then for any non-negative integer $r$ we obtain

$$
\begin{aligned}
\operatorname{div}_{C}\left(\frac{f d y}{y^{1-r}}\right)= & \left(2 n m\left(P_{1}^{\prime}\right)+r(n-2 s)-1\right) P_{1} \\
& +\left(2 n m\left(P_{2}^{\prime}\right)+r(n+2 s)-1\right) P_{2}+\left(m\left(Q^{\prime}\right)-r-1\right) \pi^{*}\left(Q^{\prime}\right) \\
& +\sum_{i=1}^{3}\left(m\left(R_{i}^{\prime}\right)+1\right) \pi^{*}\left(R_{i}^{\prime}\right)+\sum_{P^{\prime} \in S} m\left(P^{\prime}\right) \pi^{*}\left(P^{\prime}\right),
\end{aligned}
$$

where we set $S=E \backslash\left\{P_{1}^{\prime}, P_{2}^{\prime}, Q^{\prime}, R_{1}^{\prime}, R_{2}^{\prime}, R_{3}^{\prime}\right\}$. Fix $l \in\{0,1, \ldots, 2 s-1\}$, and let $r$ be a positive even integer with $(2 l n-1) /(2 s)<r \leq(2(l+1) n-1) /(2 s)$. If $f_{r} \in L\left(D_{r}^{\prime}\right)$, then

$$
\operatorname{ord}_{P_{1}}\left(\frac{f_{r} d y}{y^{1-r}}\right)=2(l+1) n-1-2 s r \geq 0
$$

and

$$
\operatorname{ord}_{P_{2}}\left(\frac{f_{r} d y}{y^{1-r}}\right)=2 s r-(2 \ln -1)-2 \geq 0 .
$$

In fact, suppose that $2 s r-(2 \ln -1)=1$, which implies that $r=\ln / s$. We know that $(n, s)=1, n$ is odd and $r$ is even. Hence $l / s$ must be even, which implies that $l=2 u s$ with a non-negative integer $u$. In view of $0 \leq l \leq 2 s-1$ we must have $l=0$, which implies that $r=0$. This is a contradiction. Hence $2 s r-(2 \ln -1)-2 \geq 0$. Therefore $f_{r} d y / y^{1-r}$ is a regular 1 -form on $C$, which implies that $2 n-(2 s r-2 n l)($ resp. $2 s r-2 n l)$ is a gap at $P_{1}\left(\right.$ resp. $\left.P_{2}\right)$.

Now we show that

$$
\begin{aligned}
\{2 s r-2 n l \mid & l=0,1, \ldots, 2 s-1, r \text { is even }>0 \\
& \text { with } \left.\frac{2 l n-1}{2 s}<r \leq \frac{2(l+1) n-1}{2 s}\right\}=\{2,4, \ldots, g-2\} .
\end{aligned}
$$


First we show that the above elements $2 s r-2 n l$ are distinct. Let $l^{\prime} \in$ $\{0,1, \ldots, 2 s-1\}$ with $l^{\prime} \geq l$ and let $r^{\prime}$ be even with

$$
\frac{2 l^{\prime} n-1}{2 s}<r^{\prime} \leq \frac{2\left(l^{\prime}+1\right) n-1}{2 s} \text { such that } 2 s r-2 n l=2 s r^{\prime}-2 n l^{\prime} .
$$

Then $n\left(l^{\prime}-l\right)=s\left(r^{\prime}-r\right)$. In view of $(n, s)=1$ we obtain $s \mid\left(l^{\prime}-l\right)$, which implies that $l^{\prime}=l$ or $l^{\prime}=l+s$. Hence we may assume that $l^{\prime}=l+s$, which implies that $r^{\prime}-r=n$. Since $r^{\prime}-r$ is even and $n$ is odd, this is a contradiction. Hence the elements $2 s r-2 n l$ are distinct.

Next if $l=0$ (resp. $l=2 s-1$ ), then

$$
\frac{2 l n-1}{2 s}=\frac{-1}{2 s}<0 \quad\left(\text { resp. } 2 n-1 \leq \frac{2(l+1) n-1}{2 s}=2 n-\frac{1}{2 s}<2 n=g\right) .
$$

In view of $r>0$ the cardinality of the set of the elements $2 s r-2 n l$ is equal to that of $\{2,4, \ldots, g-2\}$. Moreover, $1 \leq 2 s r-2 n l$. In view of $r \leq$ $(2(l+1) n-1) /(2 s)$ we have $2 s r-2 n l \leq g-1$. Hence we obtain the desired result. Therefore $2,4, \ldots, g-2$ are gaps at $P_{1}$ and $P_{2}$.

Now if $f_{0} \in L\left(D_{0}^{\prime}\right)$, then

$$
\operatorname{ord}_{P_{i}}\left(\frac{f_{0} d y}{y}\right)=2 n-1=g-1 \quad \text { for } i=1,2,
$$

which implies that $g$ is also a gap at $P_{1}$ and $P_{2}$. Let $f_{r_{1}} \in L\left(D_{r_{1}}^{\prime}-P_{1}^{\prime}\right) \neq\{0\}$. Then

$$
\operatorname{ord}_{P_{1}}\left(\frac{f_{r_{1}} d y}{y^{1-r_{1}}}\right)=4 n-2=(2 g-1)-1
$$

and

$$
\begin{aligned}
\operatorname{ord}_{P_{2}} & \left(\frac{f_{r_{1}} d y}{y^{1-r_{1}}}\right) \\
& \geq-2 n \cdot \frac{(4 s-2 q-1) n-(2 s-1)+4 s(2 s-q)}{4 s}+r_{1}(n+2 s)-1=0 .
\end{aligned}
$$

Therefore $f_{r_{1}} d y / y^{1-r_{1}}$ is a regular 1 -form on $C$, which implies that $2 g-1$ is a gap at $P_{1}$. Moreover, let $f_{r_{2}} \in L\left(D_{r_{2}}^{\prime}-P_{2}^{\prime}\right) \neq\{0\}$. Then

$$
\operatorname{ord}_{P_{1}}\left(\frac{f_{r_{2}} d y}{y^{1-r_{2}}}\right) \geq-2 n \cdot \frac{(2 q+1) n-(2 s+1)-4 s q}{4 s}+r_{2}(n-2 s)-1=0
$$

and

$$
\operatorname{ord}_{P_{2}}\left(\frac{f_{r_{2}} d y}{y^{1-r_{2}}}\right)=(2 g-1)-1 .
$$

Therefore $2 g-1$ is a gap at $P_{2}$. In the same way as in Section 4 we get $G\left(P_{1}\right)=G\left(P_{2}\right)=\{1,2, \ldots, g-2, g, 2 g-1\}$. 


\section{References}

[1] M. Coppens, The Weierstrass gap sequences of the total ramification points of trigonal coverings of $\mathbb{P}^{1}$, Indag. Math. 47 (1985), 245-276.

[2] T. Kato, On Weierstrass points whose first non-gaps are three, J. Reine Angew. Math. 316 (1980), 99-109.

[3] T. Kato and R. Horiuchi, Weierstrass gap sequences at the ramification points of trigonal Riemann surfaces, J. Pure Appl. Algebra 50 (1988), 271-285.

[4] J. Komeda, On Weierstrass points whose first non-gaps are four, J. Reine Angew. Math. 341 (1983), 68-86.

[5] - Numerical semigroups and non-gaps of Weierstrass points, Res. Rep. Ikutoku Tech. Univ. B-9 (1985), 89-94.

[6] - On the existence of Weierstrass gap sequences on curves of genus $\leq 8$, J. Pure Appl. Algebra 97 (1994), 51-71.

[7] - Non-Weierstrass numerical semigroups, preprint.

[8] I. Kuribayashi and K. Komiya, Automorphisms of a compact Riemann surface with one fixed point, Res. Rep. Fac. Educ. Yamanashi Univ. 34 (1983), 5-9.

[9] H. Pinkham, Deformations of algebraic varieties with $\mathbf{G}_{m}$ action, Astérisque 20 (1974), 1-131.

Department of Mathematics

Kanagawa Institute of Technology

Shimo-ogino 1030, Atsugi-shi

Kanagawa 243-02, Japan

E-mail: komeda@gen.kanagawa-it.ac.jp 